\title{
Controladores, tratamento de sinais e programação aplicados à piscicultura
}

\author{
Gabriel Oliveira Ferreira ${ }^{1}$, Luís Filipe Pereira Silva ${ }^{2}$ \\ 12 Engenharia Mecatrônica - Centro Federal de Educação Tecnológica (CEFET-MG) \\ Caixa Postal 400 - 91.35.503-822 - Divinópolis - MG - Brasil \\ \{gabriel, luis\}gabrielolifer95egmail.com, luis@cefetmg.br
}

\begin{abstract}
In this article is presented the development of closed loop control techniques that optimize the fish production in closed environment, by controlling the water temperature. It is possible to see that plenty of rudimentary techniques are still used in this area, which involves a lot of money, $R \$ 2.02$ billions in revenue just in 2013, according to IBGE. There is, in this project, the process automation using microcontrollers, sensors and actuators. With the system's mathematics models, that will provide enough data about the water temperature, digital controllers are calculated and programmed on the microcontroller used throughout the project, boosting the fish's growth and reproduction.
\end{abstract}

Resumo. Neste artigo é apresentado o desenvolvimento de técnicas de controle em malha fechada, visando a otimização da produção de peixes em cativeiro por meio do controle de temperatura da água onde eles estão situados. Vê-se que muitas técnicas rudimentares são aplicadas em tal meio, que circula grandes quantias de dinheiro, 2,02 bilhões apenas em 2013, de acordo com o IBGE. No projeto em questão, tem-se automação do processo por meio de microcontroladores, sensores e atuadores. Com a modelagem dos sistemas físicos que descrevem a dinâmica da temperatura da água, compensadores digitais são calculados e, então programados no microcontrolador utilizado ao longo do projeto, potencializando o crescimento e reprodução dos peixes.

\section{Introdução}

A automação está presente em diversos segmentos, sendo possível verificar sua utilização em grande escala na agricultura. De acordo com [A. Alvarenga 2014], através de sensores, atuadores e algoritmos computacionais, consegue-se aumento na prosperidade de cultivos, além de possibilitar economia de água, visto que, no Brasil, cerca de $72 \%$ desse recurso é usado para tal finalidade [C. Cremasco 2015]. Atualmente, vê-se que vários projetos sobre tanques voltados para a piscicultura são estudados, como pode ser visto em [A. Cardoso 2016] e [M. Mallasen 2012]. Isso pode ser explicado pela necessidade de otimizar a produção de peixes, buscando seu crescimento e reprodução máximos em um tempo mínimo. Há diversos estudos acerca do controle de temperatura da água em ambientes fechados, o que pode impactar diversas áreas, como em técnicas de reversão sexual dos peixes. Para maiores detalhes, veja [A. Correia 2006].

Quando se deseja controlar um sistema, uma das formas mais eficazes é colocá-lo em malha fechada com um compensador. Tendo um controlador projetado de forma adequada, garante-se estabilidade, robustez a variação de parâmetros do modelo do sistema 
e atenuação do efeito de ruídos [R. Dorf 2009]. Os controladores PID são constituídos de três ações: proporcional, integral e derivativa, sendo que as três em conjunto garantem características de regime transitório e estacionário desejadas [Ogata 2010]. Na agricultura, tal estratégia é muito utilizada para controlar a temperatura de ambientes fechados, evitando variações climáticas muito acentuadas [O. Santos 2017].

Neste artigo, propõe-se um sistema de tanques voltados para piscicultura que, a partir de controladores PID's, consiga levar a temperatura da água para $28^{\circ} \mathrm{C}$, ideal para as tilápias [Abrunhosa 2011], que, de acordo com [M. Bandeira 2017], é a espécie mais produzida no país. Para tanto, trata-se, ao longo do texto, sobre a modelagem matemática dos tanques, projeto de compensadores discretos no tempo para os mesmos e sobre os melhores atuadores e sensores para a construção de um protótipo onde os estudos podem ser testados e validados.

\section{Trabalhos Relacionados}

Recentemente, verifica-se uma grande evolução no desenvolvimento de aplicativos que auxiliam no monitoramento dos ambientes aquáticos, onde técnicas de controle são aplicadas. Por meio de softwares, é possível identificar e analisar se as grandezas estão com grau de controle satisfatório ou se alguma ação corretiva deve ser tomada. De acordo com [G1 ], a tecnologia vem ganhando cada vez mais espaço entre os piscicultores, pois "em vez de percorrer os tanques com pranchetas na mão, os tratadores agora levam tablets "e "programas de computador e aplicativos ajudam no planejamento da criação ".

Dentre as empresas que inserem tecnologia na aquicultura, é possível destacar a Aquiculture Production Technology Ltd. Ela atua em 30 países de 5 continentes e realiza todas as etapas de projeto, como controle de temperatura, $\mathrm{pH}$, quantidade de amônia, claridade da água, etc.

Em relação aos novos estudos sobre inserção tecnológica no meio da piscicultura, os mais relevantes estão sendo realizados na Europa e América do Norte. Este último, realizado no Canadá, planeja integrar as áreas de aquicultura, agricultura e energia renovável, sendo necessárias tecnologias vindas da Alemanha, Canadá e Israel.

\section{Desenvolvimento}

Os peixes são animais pecilotérmicos [P. Rebouças 2014], logo sua temperatura corporal varia de acordo com o ambiente em que estão submetidos, o que pode acarretar diversas consequências indesejadas em seu cultivo. De acordo com [A. Ostrensky 1998] "quando a temperatura da água varia, todo metabolismo do peixe é afetado. Em temperaturas mais altas, os peixes de clima quente comem mais, ficam mais ágeis, crescem mais; quando a temperatura cai, os peixes deixam de comer e diminuem bastante seu ritmo biológico ". Dentre as diversas variáveis que influenciam na qualidade da água e impactam no desenvolvimento dos peixes além da temperatura, é possível citar: $\mathrm{pH}$, oxigênio dissolvido, amônia, transparência da água, etc.[Kubitza 2011].

O funcionamento do sistema de tanques proposto segue a seguinte metodologia: tem-se água separada em três reservatórios distintos, em que no primeiro a água é controlada a $35^{\circ} \mathrm{C}$, no segundo a $28^{\circ} \mathrm{C}$ e no terceiro, o fluido está na temperatura ambiente. A água que possui maior quantidade de calor (setas vermelhas) é bombeada sempre na parte 
inferior da planta, favorecendo as trocas de calor por convecção devido à sua densidade inferior [S. Vilar 2010], conforme Figura 1. Já as setas azuis representam a água com menor quantidade de calor, possuindo maior densidade.

Caso o tanque 2 (local onde os peixes são posicionados) necessite adquirir calor, o mesmo recebe água do primeiro reservatório; caso necessite diminuir sua temperatura, recebe água do tanque 3.

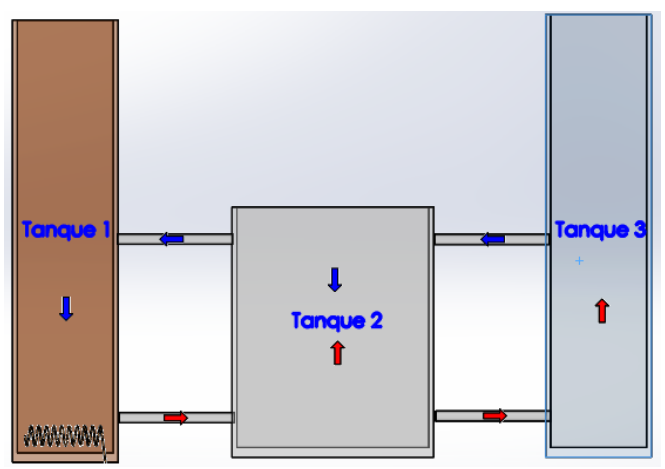

Figura 1. metodologia de circulação da água

Para realizar a medição de temperatura, usa-se o sensor DS18B20, que trabalha de $-55^{\circ} \mathrm{C}$ a $125^{\circ} \mathrm{C}$, possui resolução de $0,25^{\circ} \mathrm{C}$ e um tempo de conversão de temperatura de 187,5 milissegundos (dados obtidos a partir do datasheet do produto).

Já para possibilitar que os tanques forneçam água um para o outro, são usadas 4 bombas do modelo Brushless DC Pump, que possuem tensão de trabalho de $12 \mathrm{~V}$ e corrente nominal de $0,35 \mathrm{~A}$; suas vazões máximas são de $60 \mathrm{ml}$ por segundo. Para acionálas, utiliza-se o módulo de ponte H-L298N, visto que o mesmo consegue alimentá-las com valores de $0 \mathrm{~V}$ a $12 \mathrm{~V}$, de acordo com tensão aplicada em uma de suas entradas.

O princípio usado para controlar a vazão de saída das bombas é o PWM, em que a entrada da ponte $\mathrm{H}$ recebe $0 \mathrm{~V}$ ou $5 \mathrm{~V}$ com determinada largura de pulso, possibilitando que em sua saída tenha o valor correspondente com a mesma largura de pulso, porém com sinais de $0 \mathrm{~V}$ ou $12 \mathrm{~V}$. Variando o período em que o sinal de saída do microcontrolador está em nível lógico alto, tem-se uma faixa de tensão recebida pelas bombas (de 0V a $12 \mathrm{~V}$ ), a partir de um sinal digital [N.Tomazio 2017].

$\mathrm{O}$ atuador presente no tanque 1 é uma resistência elétrica, que possui potência de $4000 \mathrm{~W}$ e tensão nominal de $220 \mathrm{~V}$. Para seu acionamento, foi utilizado um relé de estado sólido do tipo SSR-25, que pode chavear tensões entre 24 e $380 \mathrm{~V} \mathrm{AC}$, sendo acionado apenas por $3 \mathrm{~V}$ DC.

\subsection{Modelagens}

Para o tanque 1, tem-se o seguinte cenário: a resistência elétrica cede determinada quantidade de calor por unidade de tempo (J/s), sendo que tal grandeza é absorvida pela água ou perdida por convecção entre as paredes do tanque e o meio, que se encontra em uma temperatura mais baixa, situação representada pela Equação 1. Destaca-se que os tanques são de vidro, material que possui baixa condutividade térmica, reduzindo as perdas de calor. 


$$
P_{\text {res }}=\dot{Q}_{\text {absorvido }}+\dot{Q}_{\text {convec çao }}
$$

onde $P_{\text {res }}$ representa a potência da resistência (Watts), $\dot{Q}_{\text {absorvido o calor absorvido pela }}$ água por segundo (J/s) e $\dot{Q}_{\text {convecçao }}$ o calor perdido por convecção para o meio (J/s).

Da Equação (1), pode-se chegar a Equação (2).

$$
P_{r e s}=m_{1} \cdot C \cdot \frac{\delta T}{\delta t}+h_{a r} \cdot A \cdot\left(T_{p}-T_{f}\right)
$$

onde $m_{1}$ representa a massa de água presente no tanque $1(\mathrm{Kg}), h_{a r}$ o coeficiente de convecção do ar (5-30 $\left.\frac{W}{m^{2} K}\right), A$ é a área total do tanque $\left(m^{2}\right), T_{p}$ a temperatura da superfície de vidro $\left({ }^{\circ} \mathrm{C}\right.$ ou $\left.\mathrm{K}\right), T_{f}$ a temperatura ambiente $\left({ }^{\circ} \mathrm{C}\right.$ ou $\left.\mathrm{K}\right)$ e $C$ o calor específico da água $\left(\mathrm{J} / \mathrm{Kg} .{ }^{\circ} \mathrm{C}\right)$;

Aplicando a Transformada de Laplace na Equação (2), tem-se a Equação (3):

$$
\frac{T(s)}{P_{\text {res }}}=\frac{\frac{1}{h_{\text {ar }} \cdot A}}{\frac{m_{1} \cdot C}{h_{\text {ar }} \cdot A} s+1}
$$

O processo de obtenção das equações que descrevem a dinâmica do tanque 2 se dá de forma similar à vista acima. Conforme já citado, a energia absorvida pela água nesse recipiente advém da água aquecida pela resistência elétrica, Equação (4).

$$
\dot{Q}_{q}=\dot{Q}_{2}+\dot{Q}_{\text {conveccao } 2}
$$

onde $\dot{Q}_{q}$ representa a quantidade de calor cedida pela água quente por segundo $(\mathrm{J} / \mathrm{s}), \dot{Q}_{2}$ a quantidade de calor absorvida pela água do tanque 2 por segundo $(\mathrm{J} / \mathrm{s})$ e $\dot{Q}_{\text {conveccao } 2}$ é a quantidade de calor perdida pelo tanque 2 por convecção para o meio $(\mathrm{J} / \mathrm{s})$.

Da Equação (4) pode-se inferir a Equação (5):

$$
\dot{Q}_{q}=m_{2} \cdot C \cdot \frac{\delta T}{\delta t}+h_{a r} \cdot A_{2} \cdot\left(T_{p 2}-T_{f}\right)
$$

onde $m_{2}$ é a massa de água presente no tanque $2(\mathrm{Kg}), A_{2}$ representa a área da região de contato entre água e ar $\left(m^{2}\right)$ e $T_{p 2}$ é a temperatura da água presente no tanque $2\left({ }^{\circ} \mathrm{C}\right.$ ou $\mathrm{K})$;

Aplicando a Transformada de Laplace na Equação (5), tem-se a seguinte função de transferência:

$$
\frac{T(s)}{\dot{Q}_{q}}=\frac{\frac{1}{h_{a r} \cdot A_{2}}}{\frac{m_{2} \cdot C}{h_{a r} \cdot A_{2} .} s+1}
$$

Conforme pode ser verificado nas Equações (3) e (6), a modelagem dos tanques está em função das variáveis que influenciam no processo. Esse procedimento possibilita modelar a planta para diversas dimensões. Como se trata de um protótipo, definiu-se 
medidas que possibilitassem simular o ambiente em questão, apresentando constantes de tempo relativamente elevadas, cerca de 5.700 segundos para o tanque 1 e 42.000 para o segundo (em malha aberta).

\subsection{Projeto dos controladores}

Os compensadores calculados para os dois recipientes são projetados pelo método Lugar das Raízes. Como o tanque 1 não possui um atuador que diminui a temperatura da água, seu controlador age de forma que o sistema não apresente overshoot (seus polos em malha fechada não possuem componentes imaginárias). Fixando o tempo de acomodação em 100 segundos, calcula-se a função de transferência do controlador, apresentada pela Equação (7).

$$
G_{c}(s)=\frac{312 \cdot\left(s+5,3 \cdot 10^{-3}\right)}{s}
$$

Visando embarcar o sistema e ganhos de processamento, aplica-se técnicas de Controle Digital. Sendo assim, o primeiro passo é determinar o período de amostragem do sistema; para tal, faz-se uso do Critério de Nyquist, que afirma que a frequência de amostragem deve ser maior que, no mínimo, o dobro daquela apresentada pelo sinal que se deseja amostrar [Lathi 2011]. Por conseguinte, através do diagrama de Bode, determina-se a frequência de corte da planta em malha fechada $(0,0385 \mathrm{rad} / \mathrm{s})$ e o período de amostragem adequado para discretizar a Equação (7) no tempo: 1,7 segundos.

O método de integração numérica utilizado para a discretização do controlador é o de Tustin. Com o período de amostragem e o método de integração definidos, é possível calcular a função de transferência discreta no tempo do controlador contínuo definido acima, Equação (8).

$$
G_{c}(z)=\frac{U(z)}{E(z)}=\frac{312,21 \cdot(z-0,9989)}{z-1}
$$

Para inserir a função de transferência do compensador no microcontrolador, faz-se necessário o cálculo de sua equação a diferença, Equação (9):

$$
u(k)=312,21 . e(k)-311.866 . e(k-1)+u(k-1)
$$

Sendo que k representa amostras coletadas pelo microcontrolador, u o sinal de controle e $e$ a função de erro do sistema em malha fechada.

Para o tanque 2, define-se $2 \%$ de overshoot e um tempo de acomodação de 100 segundos. Dessa forma, tem-se que água está sempre bem próxima da temperatura ideal para os peixes, $28^{\circ} \mathrm{C}$. A função de transferência do compensador projetado pode ser verificada na Equação (10).

$$
G_{c}(s)=\frac{89,67 s+0,002134}{s(s+0,08)}
$$


Assim como feito para o controlador anterior, tem-se a representação discreta no tempo da função do controlador apresentada na Equação (10), com período de amostragem de $2 s$ :

$$
C_{d}(z)=\frac{U(z)}{E(z)}=\frac{83,03 . z^{-2}+0,002134 . z^{-1}-83,03}{z^{-2}-1,852 . z+0,8519}
$$

E, a seguir, tem-se a equação a diferença da função de transferência (11).

$u(k)=83,03 . e(k)+0,003952 . e(k-1)-83,03 . e(k-2)+1,852 . u(k-1)-0,8519 . u(k-2)$

\section{Resultados}

\subsection{Tanque 1}

A partir da planta montada, instrumentada e com sensores e atuadores calibrados, é possível aplicar várias entradas no sistema e no modelo, a fim de verificar se esse descreve a dinâmica daquele. Então, aplica-se dois degraus na planta (o primeiro logo no início da simulação e o segundo com cerca de 6200 segundos); após decorridos 185 minutos, retirou-se os degraus aplicados. O mesmo procedimento é realizado no modelo matemático, sendo possível verificar o comportamento de ambos na Figura 2. Nela, constata-se resultado satisfatório, visto que as constantes de tempo das duas curvas possuem valores muito próximos, além de tenderem para os mesmos valores finais, podendose dizer que o modelo foi validado.

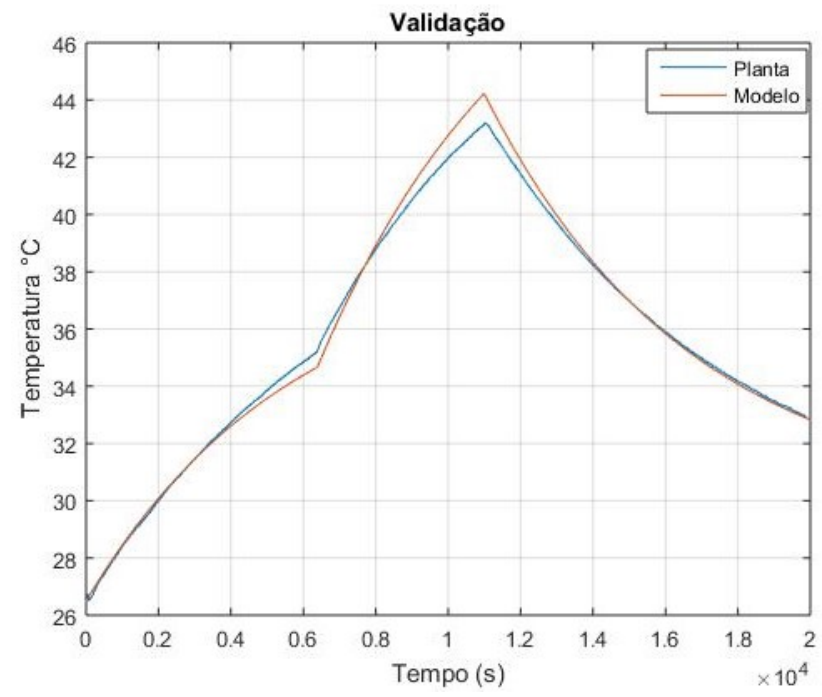

Figura 2. Validação do modelo

Entretanto, para atingir o resultado eficaz acima, é necessário realizar um ajuste fino no ganho do modelo calculado, como mostrado Figura 3. Multiplicou-se a função por 1,05, número calculado dividindo-se o maior valor da curva em vermelho pelo maior da curva em azul. 


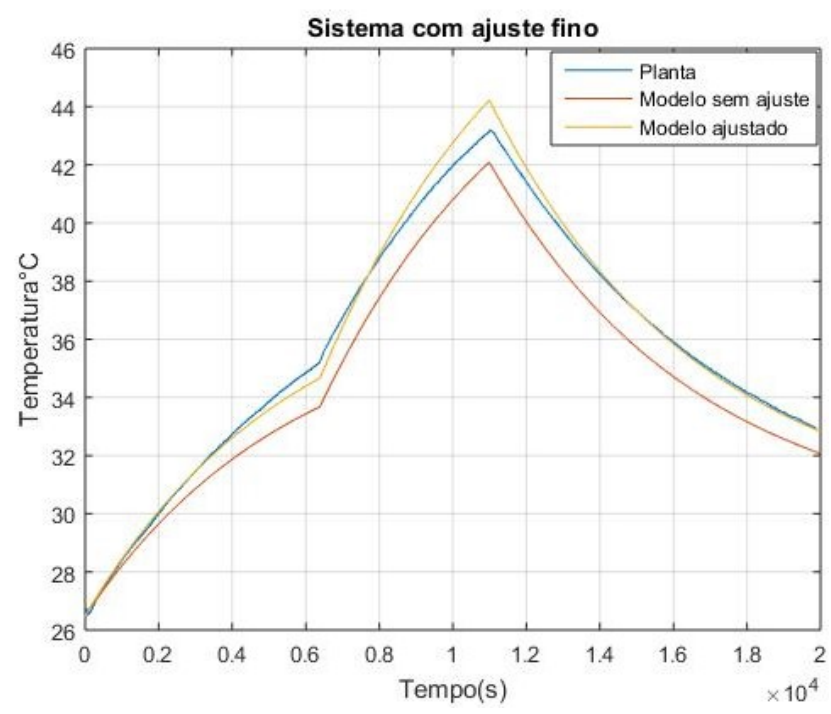

Figura 3. Ajuste fino realizado no modelo

A partir do controlador discreto em forma de uma equação a diferença, obtevese a malha fechada da planta. A partir de um degrau, obtido inserindo água em torno de $30^{\circ} \mathrm{C}$ no reservatório, é possível verificar a presença de $1,05 \%$ de overshoot e um tempo de acomodação em torno de 90 segundos, para o critério de $2 \%$, considerados resultados satisfatórios, como mostrado na Figura 4. O teste consiste em inserir diversas perturbações na planta. Como pode-se verificar na imagem, no início da simulação, a água encontra-se em torno do ponto de operação $\left(35^{\circ} \mathrm{C}\right)$, quando, de forma drástica, um líquido com temperatura mais baixa é inserida no sistema.

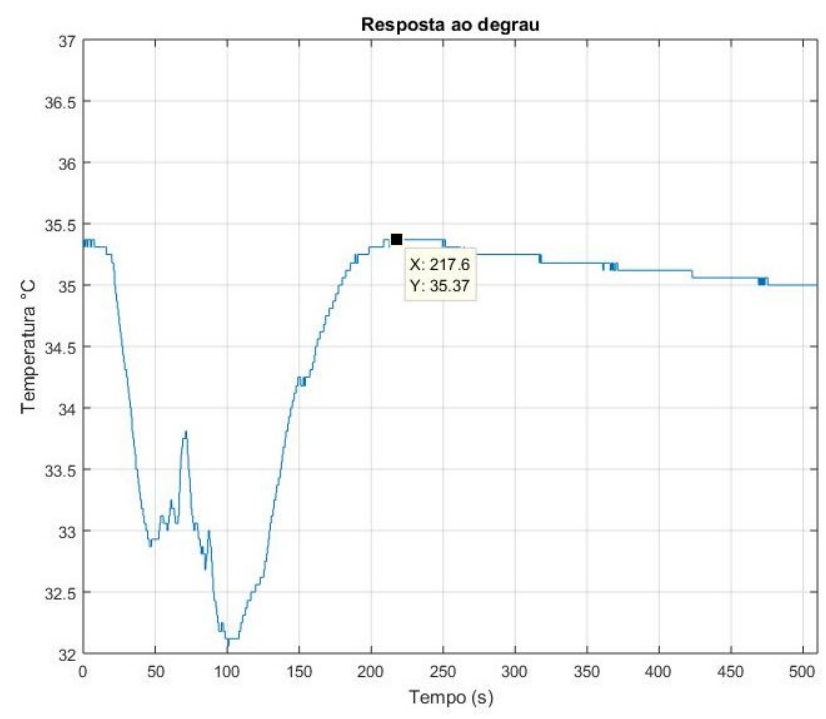

Figura 4. Planta em malha fechada com Equação a diferenças

Já na Figura 5, é apresentado o sinal de controle demandado para rejeitar as perturbações inseridas na planta. Como é possível verificar, no instante em que a temperatura da água começa a diminuir, o sinal de controle aumenta rapidamente, tornando-se menor na proporção em que o sistema aproxima-se de sua temperatura de referência, 35 
${ }^{\circ} \mathrm{C}$.

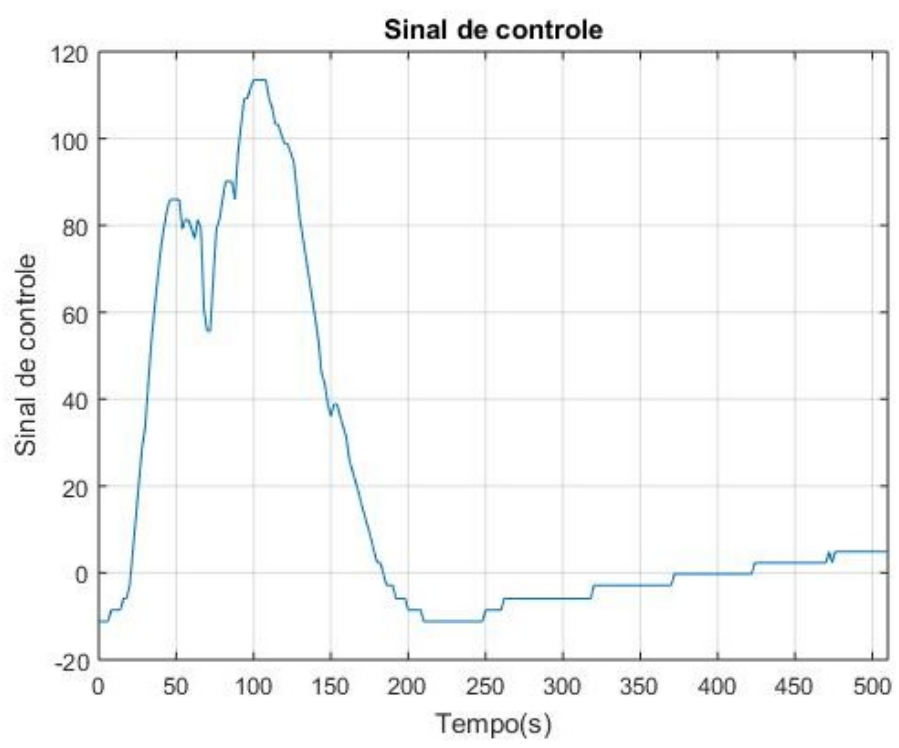

Figura 5. Sinal de controle

\subsection{Tanque 2}

Diversas entradas são aplicadas no sistema com a finalidade de validar o modelo obtido anteriormente. Os mesmos degraus aplicados na planta são aplicados na função de transferência; a resposta obtida pode ser verificada na Figura 6.

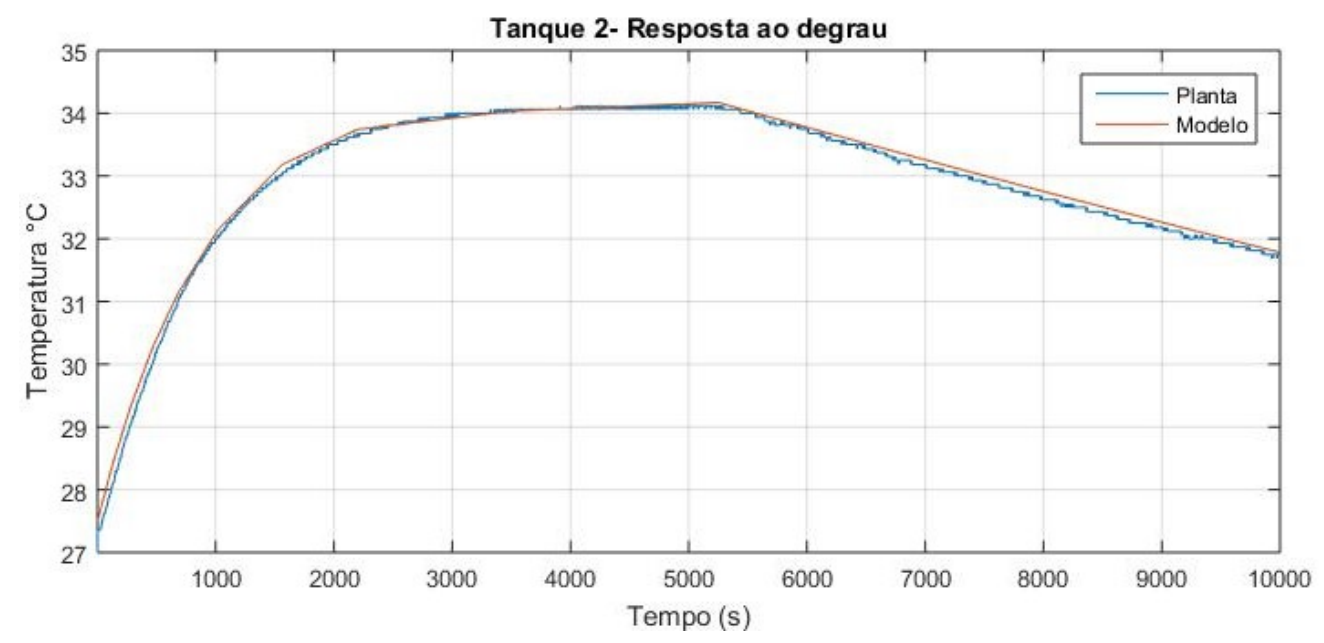

Figura 6. Validação da modelagem- Tanque 2

Através das curvas, é possível identificar que o modelo apresenta constante de tempo compatível com o sistema, bem como tende para valores finais muito próximos ao que ocorre com a planta. Outro fator importante a se destacar é a constante de tempo de descida, quando o degrau foi retirado; nesse momento (cerca de 5.200 segundos), percebe-se comportamento satisfatório do modelo, sendo possível constatar sua validação. Entretanto, é necessário realizar ajuste no ganho do mesmo, multiplicando-o por 1,2. Tem-se, na Figura 7, o modelo após ajuste comparado ao inicialmente calculado. 


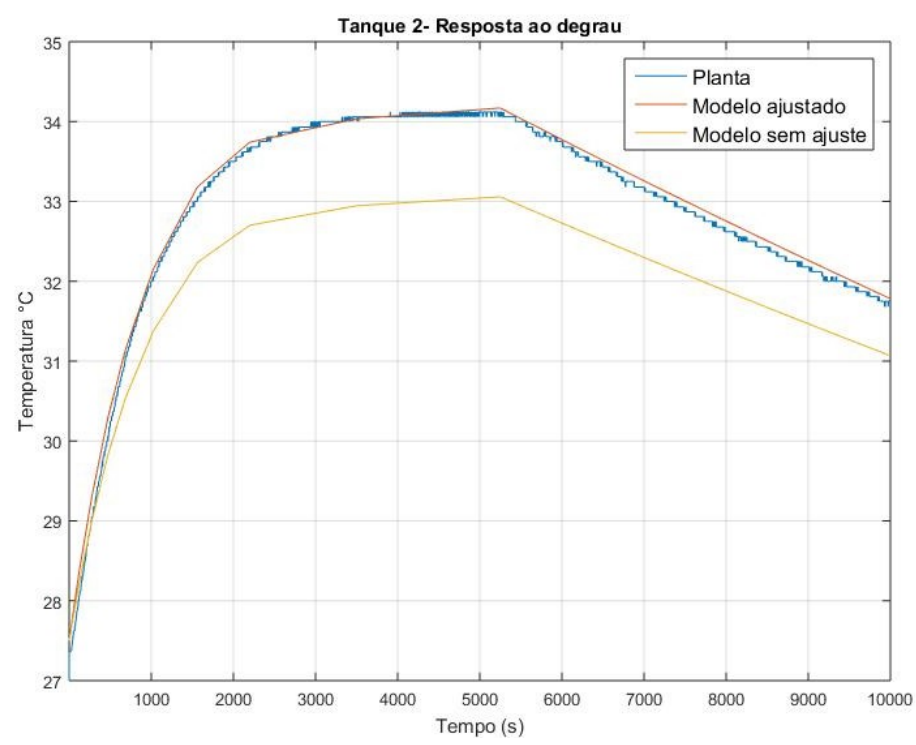

Figura 7. Comparativo entre modelos com e sem ajuste fino

Com a validação concluída, é possível colocar a planta em malha fechada com a equação a diferença calculada. Tem-se, na Figura 8, a resposta do sistema. Nela é possível observar a presença de um overshoot de 1,32 \%, dentro da margem calculada, acomodando-se em cerca de 150 segundos, para o critério de $2 \%$. O teste é realizado de acordo com a seguinte metodologia: quando o sistema estava em sua referência $\left(28^{\circ} \mathrm{C}\right)$, água com temperatura diferente de $28^{\circ} \mathrm{C}$ é inserida no mesmo.

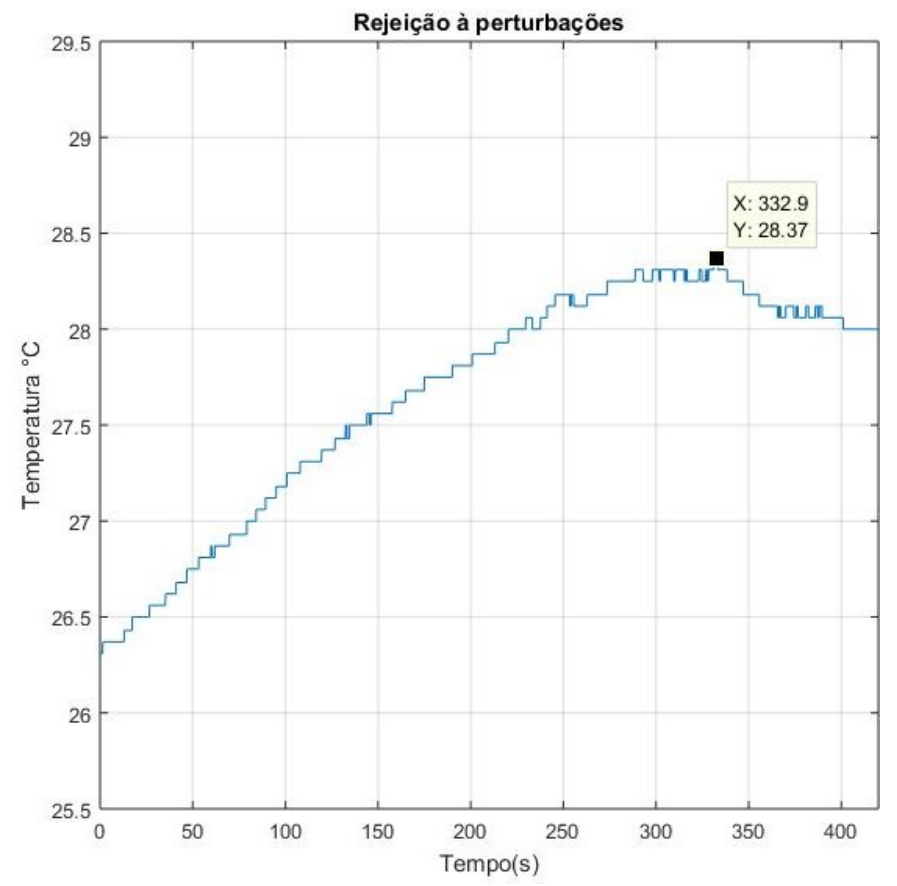

Figura 8. Sistema em malha fechada com Equação a diferenças

Já na Figura 9 tem-se o sinal de controle demandado para tal. Nela é possível 
verificar a ação do controlador, apresentando seu valor máximo quando a planta encontrase distante do ponto de operação. Com a aproximação, o sinal de controle gradativamente diminui.

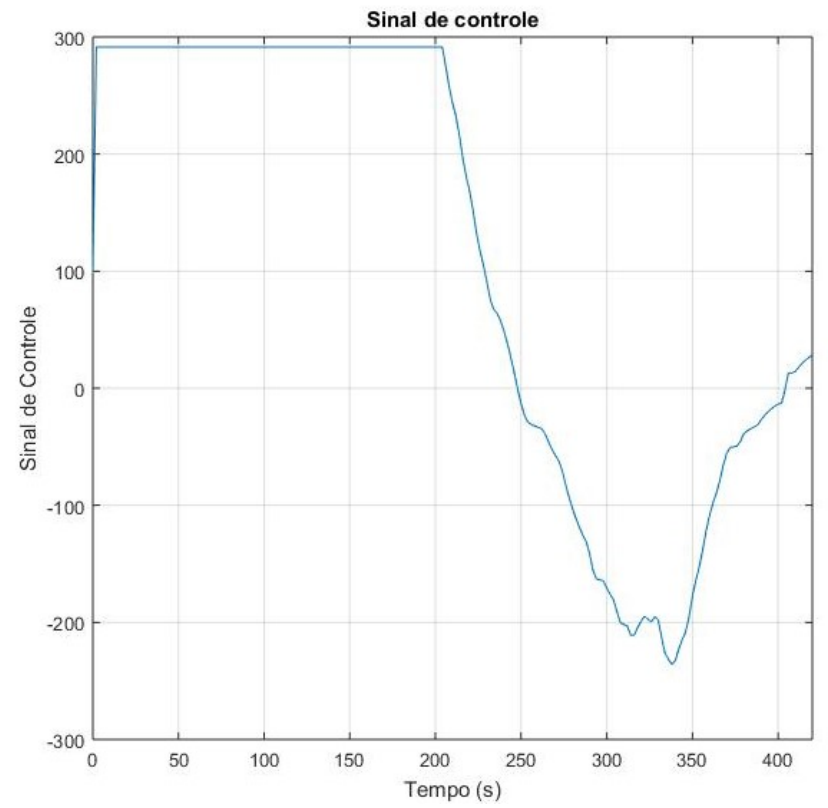

Figura 9. Sinal de controle

\section{Conclusões}

O presente artigo apresenta a definição do problema, sendo possível verificar grande investimento econômico na área da piscicultura. Nesse contexto, propôs-se o desenvolvimento de um tanque que controle a temperatura do fluido em $28{ }^{\circ} \mathrm{C}$, ideal para as tilápias. Posteriormente, definiu-se a metodologia de funcionamento da planta: como as trocas de calor seriam efetuadas, bem como os componentes eletrônicos utilizados, como sensores de temperatura DS18B20, relé de estado sólido, Arduino, resistência elétrica, ponte $\mathrm{H}$ e as bombas de água. Após tal etapa, os tanques foram modelados e tais equações foram validadas, o que permitiu o projeto dos compensadores para os dois recipientes que teriam sua temperatura controlada. Visando embarcar o sistema e ganhos com processamento, houve a discretização no tempo dos controladores segundo a aproximação de Tustin. Por fim, os compensadores foram testados diretamente na planta construída, sendo possível observar resultados satisfatórios em relação ao controle de temperatura inicialmente proposto.

A primeira sugestão de trabalhos futuros é substituição do atuador elétrico. Mesmo que a resistência consiga fornecer a potência demandada pelo sistema, há a ideia de sua troca por energia solar, via paineis solares. Dessa maneira, o custo para manter a planta em funcionamento seria drasticamente menor, além de torná-la sustentável.

Outra recomendação é a inserção de torres de resfriamento no sistema, objetivando diminuir a temperatura da água presente no Tanque 3. É válido lembrar que o fluido em nesse recipiente encontra-se à temperatura ambiente; caso o atuador seja integrado, seria possível implementar uma malha de controle no Tanque 3, deixando sua temperatura abaixo da ambiente e acelerando as trocas de calor entre os reservatórios. 


\section{Referências}

\section{Referências}

A. Alvarenga, V. Ferreira, M. F. (2014). Energia solar fotovoltaica: uma aplicação na irrigação da agricultura familiar.

A. Cardoso, S. El-Deir, M. C. (2016). Bases da sustentabilidade para atividade de piscicultura no semiárido de Pernambuco.

A. Correia, R. Moraes, J. L. e. F. S. (2006). Reversão Sexual em Larvas de Tilápia-do-Nilo em Diferentes Condições Ambientais.

A. Ostrensky, W. B. (1998). Piscicultura: fundamentos e técnicas de manejo.

Abrunhosa (2011). Piscicultura. e-Tec Brasil.

C. Cremasco, D. C. e. L. S. (2015). Sistema de automação de irrigação do plantio.

G1. http://g1.globo.com/sao-paulo/sorocaba-jundiai/nosso-campo/noticia/ 2015/05/piscicultores-investem-em-tecnologia-para-modernizar-producao. html Acesso em março de 2017.

Kubitza, F. (2011). Tilápia: tecnologia e planejamento na produção comercial. Acqua Supre, 2nd edition.

Lathi, B. (2011). Sinais e Sistemas Lineares. ARTMED EDITORA S.A., 2nd edition.

M. Bandeira, J. N. (2017). Estudo prospectivo relativo à atividade da tilápia para a indústria de alimentos no período de 2006 a 2016.

M. Mallasen, C. Carmo, A. T. (2012). Qualidade da água em sistema de piscicultura em tanques-rede no reservatório de Ilha Solteira-SP.

N.Tomazio, A.Romero, C. M. (2017). Desenvolvimento de um obturador de feixe óptico utilizando um disco rígido de computador.

O. Santos, J. Junior, M. N. (2017). Sistema de Controle de Temperatura para um Estufa com Monitoramento via Aplicativo.

Ogata, K. (2010). Engenharia de Controle Moderno. Prentice Hall, 5th edition.

P. Rebouças, L. Lima, I. D. J. F. (2014). Influência da oscilação térmica na água da piscicultura.

R. Dorf, R. B. (2009). Sistemas de Controle Moderno. 11th edition.

S. Vilar, M. Tarragó, D. B. (2010). Modelaegem da transmissão de calor por convecção: uma abordagem acessivel aos alunos de Física Geral. 